\title{
Social norms are becoming weaker
}

\author{
A study finds that social norms have become weaker in the United States over the past 200 years. The changing \\ strength of norms is linked to fluctuations in societal levels of innovation and risky behaviour.
}

\author{
Michael E. W. Varnum
}

T he United States is not what it used to be. The rules for behaviour in US society today look quite different from what they did in the late eighteenth century at the country's inception. In many ways, the United States is a far more open, tolerant and free society than it was over 200 years ago, affording far wider latitude for expression, sexual behaviour and occupational pursuits. However, previous observations about shifting norms have been largely anecdotal. Now a study by Jackson and colleagues ${ }^{1}$ published in Nature Human Behaviour provides empirical evidence that the strength of US social norms has in fact weakened over time.

To gauge the strength of norms over time, Jackson and colleagues first develop a linguistic measure, using techniques borrowed from computer science, to generate lists of words associated with the expression of tighter social norms (such as 'restrain', 'prevent', 'compel') or looser norms (such as 'allow', 'openness', 'diverse'). By applying these indices to Google's Ngram database, the authors show that the frequency of words reflecting tight social norms in US books declined over a period of 200 years, while the frequency of words reflecting looser norms increased. These changes in language are correlated with shifts in other indicators of loosening norms, such as declines in religiousness and trends in congressional legislation and cases heard by the Supreme Court, suggesting that this linguistic indicator captures meaningful changes in norm strength over time.

Having established that norms have weakened over time, Jackson and colleagues then ask whether there is a link between norm strength and a range of societal-level changes over the past five to six decades. From their central analyses, which control for monotonic effects of time, the authors report that in years when norms were weaker, more people chose relatively uncommon names for their children, and more patents, trademark applications and feature films were produced-what the authors term indicators of 'creativity' on a societal level. However, such years also had higher rates of teen pregnancy and household debt, and saw fewer teenagers attending high school-what the authors

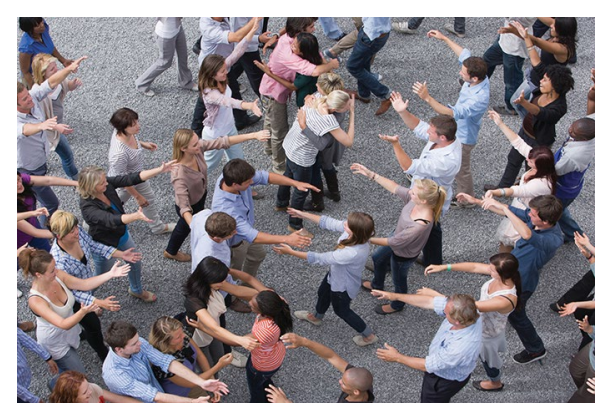

Credit: Martin Barraud/OJO Images/Getty

term indicators of 'societal order' and what others have taken as markers of a slow life history strategy ${ }^{2}$. Many of these relationships were generally strongest at a lag of a handful of years, suggesting that changes in norm strength precede these other cultural changes. The authors controlled for the effects of other variables that have been linked to these outcomes, such as the prevalence of infectious disease or gross domestic product, and for the effect of linear time trends in the data, suggesting that these relationships are robust and do not reflect spurious correlations.

Finally, Jackson and colleagues find some evidence that the strength of social norms mediates a previously observed link between levels of infectious disease and levels of conformity over time. This suggests that changing norm strength may be one mechanism by which ecological threats lead to cultural shifts. More broadly, this finding hints that there may be multiple pathways, both direct and indirect, through which changes in ecology lead to changes in culture, an idea that will have broad implications for future research and theory on cultural change.

One question unresolved by this study is what causes cultures to become looser or tighter over time. Previous cross-cultural work on social norm strength suggests that a variety of ecological threats (that is, infectious disease, war, natural disasters) are linked to stronger social norms ${ }^{3}$. To what extent levels of those threats-or other variables-are linked to changes in tightness or looseness remains an open question. Another open question is the extent to which social norms have loosened in other societies in recent decades or centuries. In many countries, this seems likely to be the case, though norm strength in other societies may have followed a more cyclical path or may have in fact increased overall during this time period. Replicating this study in a broad set of other societies could provide an even stronger test of Jackson and colleagues' key hypotheses about changes in social norms and may also provide more insight into mechanisms and moderators of these effects.

This study joins a small but growing body of research using real-world data and timeseries analysis to assess patterns of cultural change and to empirically test theories about the causes or consequences of these shifts and fluctuations ${ }^{4}$. Previous psychological research on cultural change has largely focused on shifts in values, attitudes or cognitive abilities. Jackson's team tests whether the overall strength of social norms shifts over time within a society and show that such changes can have important implications for a wide range of societal outcomes-such as teen pregnancy, household debt and educational attainment. This work should interest not only behavioural and social scientists but also policymakers. If we can understand what caused changes in levels of innovation, risky borrowing or risky sexual behaviour to occur in the past, we can also potentially predict and possibly influence these trends in the future.

Michael E. W. Varnum

Department of Psychology, Arizona State University, Tempe, AZ, USA.

e-mail:mvarnum@asu.edu

Published online: 28 January 2019

https://doi.org/10.1038/s41562-018-0519-9

References

1. Jackson, J. C., Gelfand, M., De, S. \& Fox, A. Nat. Hum. Behav. https://doi.org/10.1038/s41562-018-0516-z (2019).

2. Griskevicius, V., Delton, A. W., Robertson, T. E. \& Tybur, J. M. J. Pers. Soc. Psychol. 100, 241-255 (2011).

3. Gelfand, M. J. et al. Science 332, 1100-1104 (2011).

4. Varnum, M. E. W. \& Grossmann, I. Perspect. Psychol. Sci. 12, 956-972 (2017).

Competing interests

The author declares no competing interests. 